\title{
A MATLAB based Cellular Mobile Communication Laboratory
}

\author{
Rony Kumer Saha ${ }^{1}$ and A B M Siddique Hossain ${ }^{2}$ \\ ${ }^{1}$ Department of Electrical and Electronic Engineering, American International University-Bangladesh, Dhaka-1213, Bangladesh \\ ${ }^{2}$ Faculty of Engineering, American International University-Bangladesh, Dhaka-1213, Bangladesh \\ \{rony \& siddique\}@aiub.edu
}

\begin{abstract}
Cellular mobile communication (CMC) is one of the fastest growing sectors in telecommunication industry worldwide. The users of CMC increased rapidly in the last two decades globally. To address the high demand of CMC services, hands-on experienced workforce is a prerequisite that are primarily supposed to be provided by the universities. However, because most universities lack heavily from sufficient funding, resources, and facilities, it is difficult to setup physical laboratory of CMC to provide students with hands-on experiences. This huge investment for physical setup can be saved by developing a virtual environment using MATLAB software tools. With a soft CMC laboratory, students can model many features of $\mathrm{CMC}$, analyze and evaluate the performance in both link and system level. In this paper, we present a novel MATLAB based CMC laboratory course that comprises a total of ten experiments. The laboratory was offered in fall 2012 at American International University-Bangladesh (AIUB) for three sections each with a group of 40 students. The student's comments on understanding, achieved knowledge, overall satisfaction, importance of the laboratory course, and any changes on the existing course content are documented and assessed.
\end{abstract}

Index Terms - Cellular mobile, communication, laboratory, MATLAB, software tools.

\section{Introduction}

$\mathrm{CMC}$ is one of the rapid evolutionary technologies in the $21^{\text {st }}$ century. User demands for rich multimedia services at high data rates are ever increasing. Telecommunication vendors and operators have been consistently putting significant efforts to fulfill the user needs. Standardization bodies such as third generation partnership project (3GPP), Institute of Electrical and Electronic Engineers (IEEE) have been working towards developing next generation mobile communication standards.

As mentioned in the table I of reference [1], a number of universities have already offered laboratory courses related to the mobile wireless communication with emphasizing on hardware, software, or a combination of hardware and software. Several publications, for example [1], addressed the importance of wireless communications laboratory to complement the course while few others addressed with specific areas such as cellular network planning [2], wireless networks and mobile systems [3], wireless and mobile embedded systems [4]. However, a CMC laboratory based on MATLAB tool is not obvious. In this paper, we present a novel MATLAB based CMC laboratory that comprises a total of ten experiments as given in Table I. To limit the scope, we select six out of ten experiments to describe concisely.
However, a detailed description of all the experiments can be found by emailing at either rony@aiub.edu or siddique@aiub.edu.

The paper is organized as follows. In section II, selected six experiments of the laboratory are briefly described. The laboratory assessment is incorporated in section III. We finish the paper with a conclusion in section IV.

TABLE I: List of experiments of CMC laboratory.

\begin{tabular}{|c|c|}
\hline No & Title \\
\hline 01 & Introduction to MATLAB \\
\hline 02 & $\begin{array}{l}\text { Propagation models and path loss estimation in cellular } \\
\text { mobile communication }\end{array}$ \\
\hline 03 & $\begin{array}{l}\text { Estimation of received bit energy for data rates in wireless } \\
\text { communication }\end{array}$ \\
\hline 04 & Multipath fading in cellular mobile communication \\
\hline 05 & $\begin{array}{l}\text { Power-delay profile and Doppler spectrum for channel } \\
\text { classification in cellular mobile communication }\end{array}$ \\
\hline 06 & Design of cellular mobile system \\
\hline 07 & Estimation of bit error probability of modulation schemes \\
\hline 08 & $\begin{array}{l}\text { Radio resource allocations and scheduling in cellular mobile } \\
\text { communication }\end{array}$ \\
\hline 09 & Design of inland digital microwave link \\
\hline 10 & Experiment 10: Design of satellite link \\
\hline
\end{tabular}

\section{Laboratory Experiments}

In this section, the selected six experiments are described in terms of major objectives and significance, necessary mathematical expressions and illustrations, and relevant performance analysis and evaluations.

\section{A. Experiment 1: Propagation models and path loss} estimation in cellular mobile communication.

In this experiment, we primarily carry out the impact of carrier frequency $(f)$ and distance $(d)$ on path loss. In addition, a sensitivity analysis is carried out that provides critical parameters such as base station (BS) antenna height $\left(h_{b}\right)$, mobile station (MS) antenna height $\left(h_{m}\right)$ is incorporated for the system design and planning purpose.

We consider the very optimistic Free-space model, the very pessimistic ITU-R model, and the more realistic Hata 
model. All models are conceptually and analytically described, followed by respective simulation performance evaluation. We finish this experiment with a comparison of these path loss model simulation results as shown in fig. 1 .

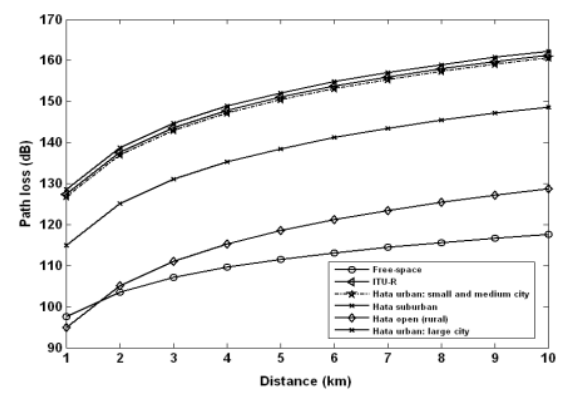

Fig.1 Path loss v ersus distance estimation $\left(f(M H z)=1800 ; h_{m}(m)=3\right.$, $h_{b}(m)=50, d(\mathrm{~km})=1$ to 10$)$.

\section{B. Experiment 2: Multipath fading in cellular mobile communication.}

In this experiment, students simulate the small-scale multipath fading effect on the transmitted signal. For non lineof-sight (NLOS) case, the received signal envelope follows Rayleigh distribution, and hence, the fading effect is simulated using Rayleigh fading function rayleighchan(). However, when at least one LOS component is present, the received signal envelope follows Rician distribution, and hence, the fading effect is simulated using Rician fading function ricianchan().

The fading effect is investigated under both frequency-flat and frequency-selective channel conditions in order to understand the effect of multipath propagation over the single path. The effect of change in Doppler spread, symbol duration, and the Rician $\mathrm{K}$-factor on the channel response is also analyzed. Fig.2 shows an example of Rayleigh fading as well as Rician fading channel responses for frequency selective fading.

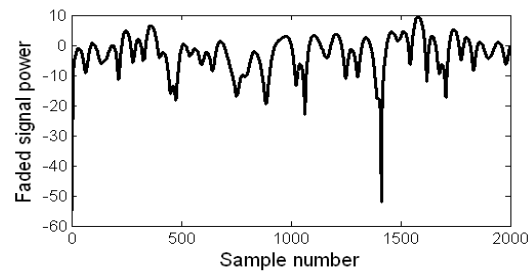

(a)

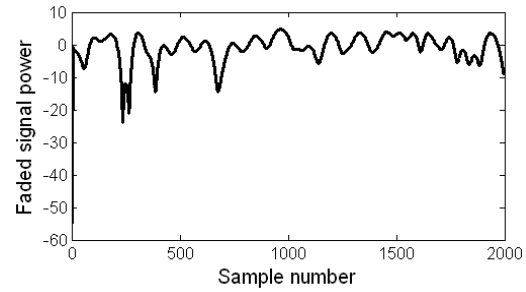

(b)

Fig.2 frequency selective (a) Rayleigh and (b) Rician Fading channel response.
C. Experiment 3: Power-delay profile and Doppler spectrum

for channel classification in cellular mobile

communication.

This experiment provides students with how to classify channels by evaluating power-delay profile and Doppler spectrum in cellular mobile communication. Power-delay profile and Doppler spectrum are major indicators for designing channel bandwidth $\left(B_{s}\right)$ and transmitted symbol duration $\left(T_{s}\right)$.

In multipath channels, the transmitted signal propagates over various paths, and each path is characterized with relative power and delay. The power-delay profile of the channel is an indication of the expected degree of dispersion of the transmitted signal that defines the maximum possible transmission bandwidth $\left(B_{c}\right)$ by evaluating delay spread of the channel for proper reception.

Similarly, the spectral broadening or Doppler spread $\left(f_{d}\right)$ of the transmitted signal, caused by the degree of relative motion between BS and MS, defines the maximum possible transmitted symbol duration $\left(T_{c}\right)$. Based on the relative magnitude of $B_{c}$ over $B_{s}$ and $T_{c}$ over $T_{s}$, the channel can be classified as given in Table II.

TABLE II: Channel classification in mobile communication.

\begin{tabular}{|c|c|c|}
\hline \multirow{2}{*}{ Channel classification } \\
\hline Parameter & Condition & Channel type \\
\hline \multirow{2}{*}{ Delay spread } & $B_{c}>>B_{s}$ & Frequency flat \\
\cline { 2 - 3 } & $B_{c}<B_{s}$ & Frequency selective \\
\hline \multirow{3}{*}{ Doppler spread } & $T_{c}>>T_{s}$ & Slow fading \\
\cline { 2 - 3 } & $T_{c}<T_{s}$ & Fast fading \\
\hline
\end{tabular}

\section{Experiment 4: Design of cellular mobile system.}

This experiment gives the students an overview on cellular mobile system design. With changing system scenario, students are able to understand how the system design parameters and requirements change for optimal performance.

The design of cellular mobile system depends on several issues. Hence, we restrict the scope of system scenario considerations to carrier-to-interference level, cell structure, system and channel bandwidths, environmental profile, cell splitting and sectorization strategy, receiver filter characteristics, traffic distribution, trunked system nature, and coverage area.

Given such an explicit system scenario, students are able to estimate system design requirements and parameters such as frequency reuse factor, minimum co-channel cell reuse ratio, spectral efficiency, system capacity, minimum adjacent channel frequency separation, number of cells required for the coverage area, channel allocations with or without sectorization, new transmit power after cell splitting, traffic intensity, bit transfer capacity, and trunking efficiency [5].

E. Experiment 5: Estimation of bit error probability of modulation schemes.

In this experiment, we are primarily concerned with 
finding an appropriate modulation scheme at the transmitting side using bit error probability measurement. We consider modulation schemes such as $M-P A M, M-P S K, M-Q A M$ and $M$ point orthogonal signal sets. We then draw a conclusion, based upon the results obtained from these considered schemes, on selecting an appropiate modulation scheme that can be well suited in power-limited region and bandwidthlimited region.

The followings (Figs. 3 and 4) are example line graphs for bit error probability versus $\frac{E_{b}}{N_{O}}$, and it can be found that irrespective of the degree of modulation, $P A M$ is susceptible more to bit errors than PSK, QAM and orthogonal signal sets. In addition, increase in the degree of modulation results in more bit errors for $P A M, P S K$, and $Q A M$. However, the opposite is the case for orthogonal signal sets. Overall, PAM and $Q A M$ schemes are bandwidth-efficient, and orthogonal signal sets are power-efficient [6].

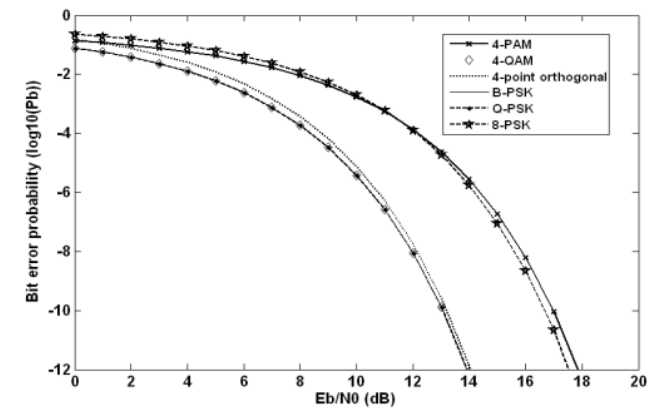

Fig.3: Bit error probability for 4-PAM, $2 \times 2$ QAM and 4-point orthogonal signal sets.

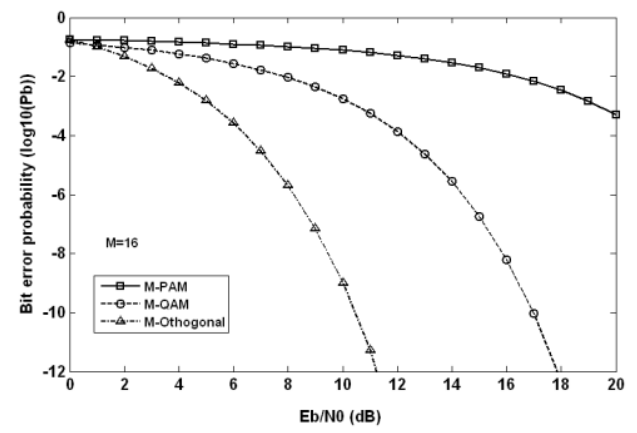

Fig.4: Bit error probability for $16-P A M, 4 \times 4 Q A M$ and 16-point orthogonal signal sets.

\section{F. Experiment 6: Design of inland digital microwave link.}

In this experiment, students study the basic concepts of high frequency wave propagation, optical and radio horizon, ducting phenomenon, Fresnel zones, earth bulge, and factor $k$ : a ratio of effective earth radius to the true earth radius and its effect on the link range. Fig.5 shows an example point-topoint microwave backhaul between base station transceivers (BTSs) $A$ and $B$ with gain and loss in different parts.

Given that transmit power is $0 \mathrm{dBW}$, total cable loss is 4 $\mathrm{dB}$, carrier frequency is $6 \mathrm{GHz}$, intermediate frequency bandwidth of the receiver is $10 \mathrm{MHz}$, receiver is at room temperature, and $90 \%$ radio link reliability, students can estimate the effective

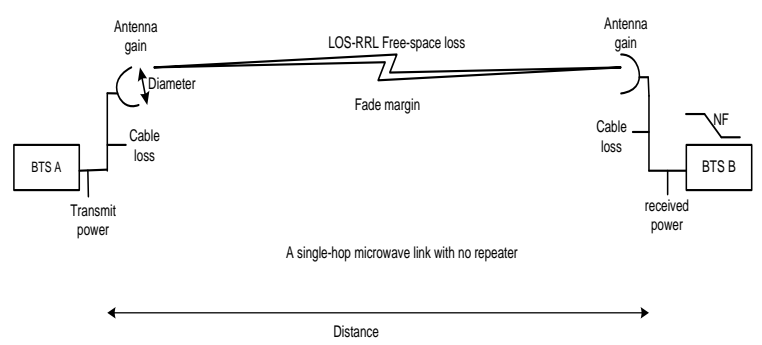

Fig.5: A typical single hop microwave communications link.

isotropic radiated power $(E I R P)$, free-space path loss of the hop, power flux density at the receiver front end, receiver noise threshold, antenna aperture diameter, and the received power. Fig. 6 is a simulated result for a single hop microwave link. All gains and losses are shown along with the signal propagation from transmitter to receiver.

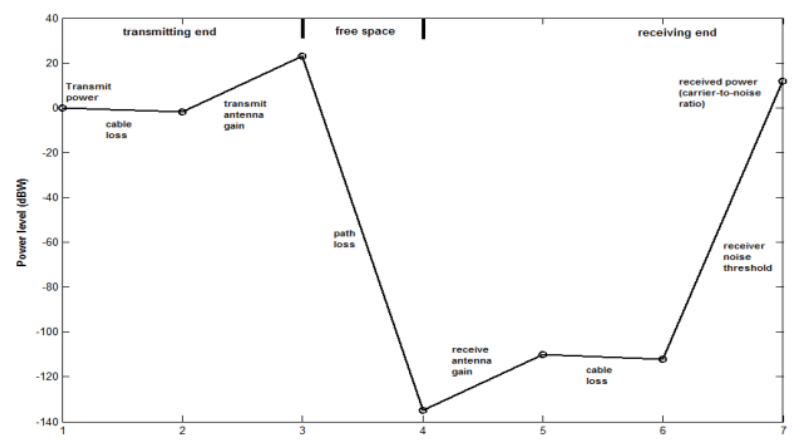

Fig.6: Typical link budget estimation of a LOS microwave link.

\section{Laboratory Assessment}

In order to assess the significance of the CMC laboratory, we carried out a student survey within the registered students of the CMC laboratory of fall 2012 semester at AIUB. The total number of students participated in the survey was ninety one. The survey questions were categorized into two parts: part I incorporates general questions and part II incorporates query based questions, with five questions in each part (Table III). Students were asked to put a tick on either Yes or No, with an option for making comments, explaining the reasons for their opinions.

From the Figs.7 (a) and (b), it can be found that overall most of the students responded to Yes. Particularly, majority have agreed upon questions 01 through 07 , i.e. the laboratory is relevant and helpful for understanding the CMC course, provides sufficient realization of the practical aspects of CMC, and is helpful for further research and professional career development in mobile communication. In addition, according to the majority of the students, the experiments have been 
written properly with easy to understand features, and the lab is equipped with sufficient resources for simulation.

TABLE III: Student survey questions of CMC laboratory assessment.

\begin{tabular}{|c|c|}
\hline No & Question \\
\hline \multicolumn{2}{|r|}{ Part I: General category } \\
\hline Q01 & $\begin{array}{l}\text { The laboratory experiments are relevant and helpful for } \\
\text { understanding the contents of the course cellular mobile } \\
\text { communication. }\end{array}$ \\
\hline Q02 & $\begin{array}{l}\text { The experiments of the lab are easy to understand and have } \\
\text { been written properly with sufficient details. }\end{array}$ \\
\hline Q03 & $\begin{array}{l}\text { The lab provides sufficient realization of the practical } \\
\text { aspects of cellular mobile communication using MATLAB } \\
\text { software tool. }\end{array}$ \\
\hline Q04 & $\begin{array}{l}\text { The lab is helpful for further research and professional } \\
\text { career development in mobile communication. }\end{array}$ \\
\hline Q05 & The lab is equipped with sufficient resources for simulation. \\
\hline \multicolumn{2}{|r|}{ Query based } \\
\hline Q06 & $\begin{array}{l}\text { Is the laboratory time of three hours duration sufficient for } \\
\text { the experiments? }\end{array}$ \\
\hline Q07 & $\begin{array}{l}\text { Is there any prerequisite the students should complete } \\
\text { before they enroll for the lab? }\end{array}$ \\
\hline Q08 & $\begin{array}{l}\text { Should there be any changes in the content, strategy, } \\
\text { methodology, etc. of the lab? }\end{array}$ \\
\hline Q09 & $\begin{array}{l}\text { Is there any deficiency in the lab experiments that should be } \\
\text { addressed? }\end{array}$ \\
\hline Q10 & Is there any comment on improving the lab? \\
\hline
\end{tabular}

Fig.7 shows the response of the students for each question of the survey (Table III). From the Fig.7 (b), for query based questions, 08 to 10, majority of the students responded almost equally to Yes or No. More specifically, the laboratory time is sufficient, and the students should complete prerequisites such as digital signal processing, telecommunications engineering, and how to work with MATLAB.

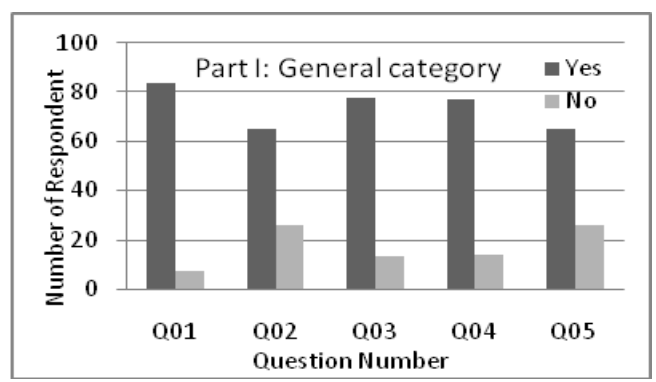

(a)

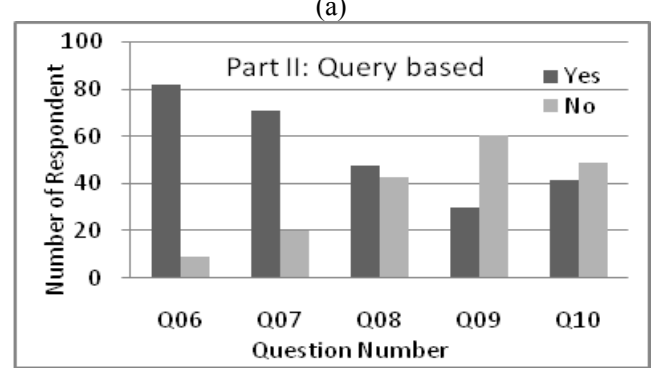

(b)

Fig.7: Response of the students to the survey questions (a) general category (b) query based.
Moreover, in the final question, students were asked to make comments on improving the laboratory. Selected few comments made by a considerable number of students are as follows: "arrangement of few field visits to telecommunication industry may facilitate students gaining some real life experiences", "few practical types of equipment can be introduced", and "understanding of MATLAB coding should be a prerequisite".

\section{Conclusion}

In this paper, we presented a novel MATLAB based cellular mobile communication (CMC) laboratory course which had been offered in fall 2012 at AIUB, Bangladesh. The laboratory course comprises of ten experiments of which six are described concisely, covering the fundamental design parameters, considerations, and estimations of CMC both in the radio interface and core network levels. In the radio interface side, estimation of path loss, fading, power delay profile, and received bit error probability, while in the core network side, estimation of link budget of earth-satellite-earth communication, inland microwave communication, and radio resource allocation and scheduling have been included. In addition, a fundamental to the MATLAB was also introduced at the very beginning for allowing students to understand of how to code in MATLAB.

A survey over ninety one students was carried out to assess the importance and impact of the laboratory. Almost all students found the laboratory experiments relevant and helpful for understanding the CMC theory course and for further research and professional career development. Since the laboratory is just MATLAB software based, the laboratory can be offered at affordable cost to provide students to gain practical realization of the CMC theory course at the university. The authors would be pleased to share the laboratory course materials with any individual or institution interested in it and can be reached at either rony@aiub.edu or siddique@aiub.edu.

\section{References}

[1] A. F. Cassara, "Wireless communications laboratory," IEEE Trans. on Educ., vol.51, no.3, pp. 132-140, Feb. 2006

[2] Z. Dawy et al., "A wireless communications laboratory on cellular network planning," IEEE Trans. on Educ., vol.53, no.4, pp. 653-661, Nov. 2010.

[3] S. F. Midkiff, "An experiential course in wireless networks and mobile systems," IEEE CS and IEEE ComSoc, pp. 9-13, Jan.-Mar. 2005.

[4] J. Chenard, et al., "A laboratory setup and teaching methodology for wireless and mobile embedded systems," IEEE Trans. on Educ., vol.49, no.1, pp. 378-384, Aug. 2008.

[5] T. S. Rappaport, Wireless communications principles and practice, $2^{\text {nd }}$ ed., NJ: Pearson Education International, 2002.

[6] P. Saengudomlert. (2011 Jan.). Digital communications. Asian Institute of Technology (AIT). Bangkok, Thailand. [online].Available: http://www.tc.ait.ac.th/faculty/poompat/. 